\title{
Nonmonotonic Stress Relaxation after Cessation of Steady Shear Flow in Supramolecular Assemblies
}

\author{
Jan Hendricks@, ${ }^{1, *}$ Ameur Louhichi, ${ }^{2,3,}$ Vishal Metri, ${ }^{4}$ Rémi Fournier, ${ }^{5}$ Naveen Reddy $\odot,{ }^{6}$ Laurent Bouteiller, ${ }^{7}$ \\ Michel Cloitre $\odot{ }^{5}$ Christian Clasen $\odot,{ }^{1}$ Dimitris Vlassopoulos $\odot,{ }^{2,3, \dagger}$ and W. J. Briels $\oplus^{4,8, \$}$ \\ ${ }^{1}$ Department of Chemical Engineering, KU Leuven, 3001 Leuven, Belgium \\ ${ }^{2}$ Institute of Electronic Structure and Laser, FORTH, P.O. Box 1527, 70013 Heraklion, Crete Greece \\ ${ }^{3}$ Department of Materials Science and Technology, University of Crete, Voutes Campus, 70013 Heraklion, Crete Greece \\ ${ }^{4}$ Computational Chemical Physics, Faculty of Science and Technology, and MESA+Institute for Nanotechnology, \\ University of Twente, P.O. Box 217, 7500 AE, Enschede, Netherlands \\ ${ }^{5}$ Molecular, Macromolecular Chemistry, and Materials, ESPCI Paris, CNRS, PSL University, 75005 Paris, France \\ ${ }^{6}$ Faculty of Industrial Engineering, Hasselt University, Martelarenlaan 42, 3500 Hasselt, Belgium, \\ and IMO-IMOMEC, Hasselt University, Wetenschapspark 1, 3590 Diepenbeek, Belgium \\ ${ }^{7}$ Sorbonne Université, CNRS, IPCM, Equipe Chimie des Polymères, 75005 Paris, France \\ ${ }^{8}$ ICS 3, Forschungszentrum Jülich, Wilhelm-Johnen-Straße, 52428 Jülich, Germany
}

(Received 18 February 2019; revised manuscript received 27 May 2019; published 20 November 2019)

\begin{abstract}
Stress relaxation upon cessation of shear flow is known to be described by single-mode or multimode monotonic exponential decays. This is considered to be ubiquitous in nature. However, we found that, in some cases, the relaxation becomes anomalous in that an increase in the relaxing stress is observed. Those observations were made for physicochemically very different systems, having in common, however, the presence of self-associating units generating structures at large length scales. The nonmonotonic stress relaxation can be described phenomenologically by a generic model based on a redistribution of energy after the flow has stopped. When broken bonds are reestablished after flow cessation, the released energy is partly used to locally increase the elastic energy by the formation of deformed domains. If shear has induced order such that these elastic domains are partly aligned, the reestablishing of bonds gives rise to an increase of the overall stress.
\end{abstract}

DOI: $10.1103 /$ PhysRevLett.123.218003

Many experiments and simulations with soft materials ranging from polymer melts or solutions to colloidal suspensions show that once an initially imposed shear rate is set to zero the stress relaxes monotonically via one or more distinct exponential processes, reflecting successively the fast retraction of small-scale, shear-induced, nonequilibrium structures and the much slower relaxation of structures induced at the larger scales [1-5]. Often out-ofequilibrium systems such as colloidal glasses, pastes, and gels do not fully relax their stresses, but exhibit residual stresses because the induced large-scale structures cannot find relaxation pathways and remain permanent in the system [6-8]. Nonetheless, a common generic feature is the monotonic decrease of the stress to its final value. At first glance this appears to be the expected behavior, since no work that would be able to raise the stress is performed on the system. However, this scenario may not always hold. Indeed, for a particular supramolecular polymer system, in which bonds were broken during steady shearing, we have found [9] a nonmonotonic relaxation of stress following cessation of shear flow, as schematically shown in Fig. 1. In order to prove the generic character of our findings, we have investigated two more physically and chemically very

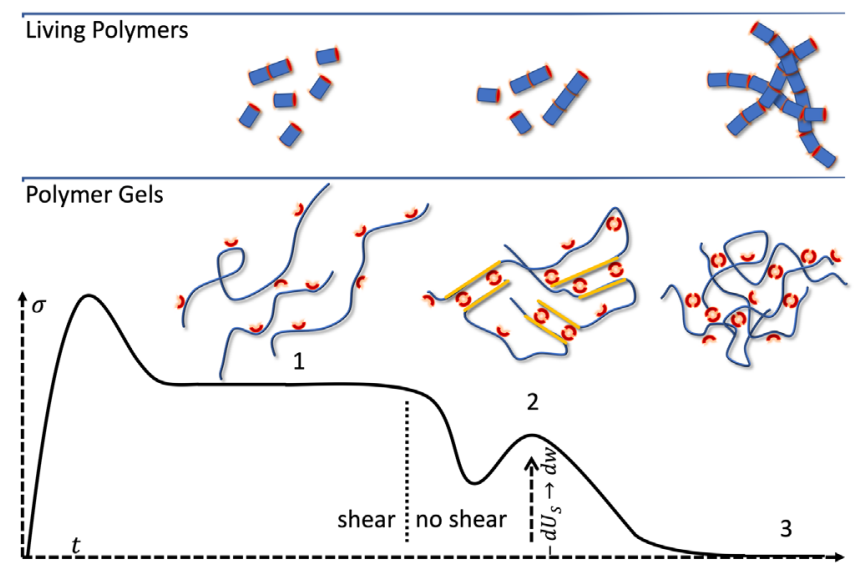

FIG. 1. Illustration of the experimental protocol and structural changes of the investigated living polymers (system A) and polymer gels (systems B and C) under shear and during relaxation. Large shear magnitudes lead to bond breakage resulting in stored energy (1). Bond reformation leads to work performed on the polymers and causes local elastic inhomogeneities, indicated by orientationally correlated local segments marked by yellow lines, resulting in increasing bulk stress (2), which eventually relaxes thermally until the equilibrated networks of living polymers or polymer gels are reestablished (3). 
different systems with different associating mechanisms $[10,11]$. All cases have in common that bonds are disrupted by subjecting the systems to steady shear flow and reform after flow cessation. We argue below that a redistribution of the released bond energy during stress relaxation gives rise to a temporary stress increase. Hence, the purpose of this Letter is to present and rationalize this counterintuitive nonmonotonic stress relaxation upon cessation of steady shear flow. Nonmonotonic stress evolution has, of course, been observed in driven systems, during startup with stress overshoots, and in banding systems where even stress undershoots may occur during the development of the bands [12-14].

The first system (A) examined is an organogel consisting of 2,4-bis (2-ethyl-hexyl-ureido) toluene (EHUT) [15,16] dissolved in dodecane, an apolar solvent. The EHUT bis-urea-based monomers self-assemble above a certain concentration through the formation of hydrogen bonds between the urea groups. Data presented in this Letter were obtained in the tube region of the phase diagram (with cross-sectional diameter of three EHUT monomers or $\sim 2.6 \mathrm{~nm}$ ) [17] at a concentration of $12 \mathrm{~g} \mathrm{~L}^{-1}$, where the tubes are long enough to entangle and exhibit a rich viscoelastic response.

The second system (B) comprises aqueous solutions of a synthetic polymer having a molar mass of $500 \mathrm{~kg} \mathrm{~mol}^{-1}$, with 5\% of the monomers being functionalized with ligands [18]. Polymer solutions with concentrations corresponding to the semidilute regime $(1.35 \mathrm{wt} \%)$ were mixed with a stoichiometric concentration of metal ions to ligands. The added iron chloride results in strong metalligand bonded supramolecular hydrogels, with up to three ligands bonded to a single metal ion.

The third system (C) consists of two complementary polymers dissolved at low concentrations $(0.35 \mathrm{wt} \%$ each) in $0.1 M$ aqueous $\mathrm{NaOH}$ at $p \mathrm{H}=12.5$ [11]. The first polymer is a partially hydrolyzed polyacrylamide grafted with phenylboronic acid moieties (HPAM-g-PBA) with a molar mass of $780 \mathrm{~kg} \mathrm{~mol}^{-1}$, and the second polymer is a fully saponified poly(vinylalcohol) (PVA) with a molar mass of $125 \mathrm{~kg} \mathrm{~mol}^{-1}$. The phenylboronic acid moeities and the 1,3 diols form phenylboronic esters, which act as reversible covalent bonds. Despite the low value of the equilibrium constant $\left(K<10 \mathrm{~L} \mathrm{~mol}^{-1}\right)$, gels are obtained at polymer concentrations in the semi-dilute unentangled regime below 2 wt $\%$ due to synergistic effects of multiple associations.

Details about rheological measurements are found in the Supplemental Material (SM) [19]. Figure S1 of the SM presents the linear viscoelastic spectra of the three systems. The range of shear rates applied before flow cessation corresponds to the frequency region associated with the breaking time of associations (above the minimum in $G^{\prime \prime}$ ) and justifies the induced orientation at steady state (Fig. 1).

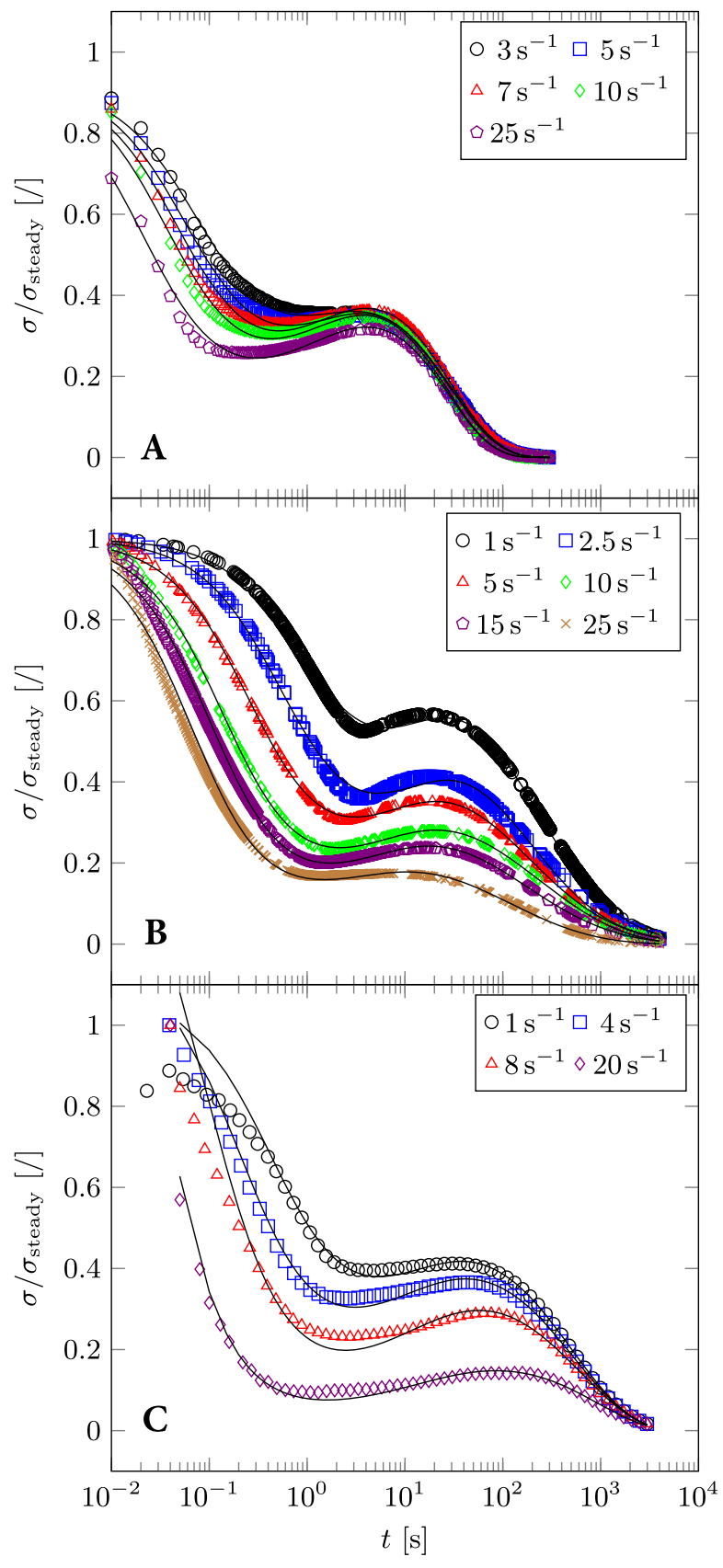

FIG. 2. Stress relaxation experiments with the supramolecular systems A (top), B (middle), and C (bottom). At intermediate times stresses increase. The shear stress is normalized to the steady-state shear stress, and shear rates applied prior to cessation of flow are listed in the legends.

Experimental stress relaxation curves after flow cessation are shown in Fig. 2 for all three systems and for different initially imposed shear rates. Time zero was set when the steady shear flow was stopped, and stresses have been normalized by their values at time zero. In all cases the stress relaxation is clearly nonmonotonic. Stresses in system A have already decayed substantially at the earliest times shown in the plot. This is because early relaxation of 
small species (see also the illustration in Fig. 2) is too fast to be resolved [9]. We note that all stresses relax eventually to zero at times of the order of the terminal time of the linear stress relaxation modulus $G(t)$, which is the Fourier transformation of $G^{\prime}$ and $G^{\prime \prime}$ [19].

Repeated runs of all experiments with newly prepared batches of the various systems confirmed our findings. Additional support from birefringence measurements and an analysis of possible motor drift (see SM [19]) confirm that the effect, although unexpected, is real. The fact that birefringence follows the stress upswing (Fig. S3 in SM) implies local orientation (see also Fig. 1). The main reason for not expecting an upswing of an initially decaying stress is the possible disagreement with either of the two laws of thermodynamics. We will now argue that this is not the case and suggest a very simple, highly approximate phenomenological model to describe our data.

In general, the microscopic state of the system is described by local strains, local order tensors, and local bond densities. The steady-state values of strain rate $\dot{\gamma}$ and total strain $\gamma$ fix the initial values for these variables. A set of evolution equations then allows the calculation of these variables at later times. The evolution equations should naturally contain random contributions obeying appropriate fluctuation-dissipation theorems. Here, we restrict ourselves to the more modest task of describing only the stress relaxation after the shear has stopped, thereby eliminating the need to include random contributions to the evolution equations. We assume that local strains and local order basically describe the same aspect of our systems, and focus on strains. Since stress is an increasing function of strain, we replace stresses by strains in the list of state variables. Although much of our argumentation is about local stresses, our final model uses only global properties of the system.

Our considerations suggest the following set of evolution equations:

$$
\begin{aligned}
d f & =-r f d t \\
d \sigma_{x y} & =-\sigma_{x y} \frac{d t}{\tau}-\sigma_{x y} B d f .
\end{aligned}
$$

Here, $f$ is the fraction of all bonds that are broken and $\sigma_{x y}$ is the measured shear stress. The coordinate frame is such that the imposed steady shear flow was in the $x$ direction with its gradient in the $y$ direction. Both $f$ and $\sigma_{x y}$ depend explicitly on time with increments $d f$ and $d \sigma_{x y}$ during a time interval $d t$. The parameters $r, \tau$, and $B$ depend implicitly on time through their dependence on $f$. The first equation tells us that the fraction of broken bonds decays with rate $r$. The second equation indicates that the stress decays at a timescale $\tau$ due to erratic motions in the system, while it increases when bonds are reestablished; with $d f$ being negative, the effective bond contribution to the stress $B$ must be positive in order to describe stress increase.

We consider the system to be isolated immediately after shearing has been stopped and describe how energy is redistributed while bonds are reestablished and the system relaxes to equilibrium. The bond energy density $U_{B}$ at any instant of time may be written as $U_{B}=(1-f) \Phi$, with $f$ being time dependent. At equilibrium, $f=0$ and the bond energy density equals $\Phi$. Notice that with this definition $\Phi$ is negative, and that by breaking bonds an energy density of $U_{S}=-f \Phi$ has been stored into the system. The total energy density $U_{\text {tot }}$ reads

$$
U_{\mathrm{tot}}=U_{T}+U_{E}+U_{B},
$$

where $U_{T}$ is the thermal energy density, i.e., the part of the energy density that depends only on temperature, and $U_{E}$ the elastic energy density, i.e., the part of the energy density that depends both on temperature and on (local) strain. Since we are considering an isolated system, the total energy density will remain constant during the relaxation process, while the individual contributions depend on time.

During the approach to equilibrium after cessation of flow, the fraction of broken bonds gradually decreases from its initial value $f_{0}$ to zero at long times, while concomitantly the stored energy decreases to zero. We assume that the released binding energy will be partly delivered as thermal energy and partly as elastic energy. During a small time interval, therefore, we may write

$$
\begin{aligned}
& d U_{B}=-\Phi d f, \\
& d U_{T}=d U_{\mathrm{err}}+(1-\alpha) \Phi d f, \\
& d U_{E}=-d U_{\mathrm{err}}+\alpha \Phi d f,
\end{aligned}
$$

where $\alpha$ denotes the fraction of binding energy released as elastic energy. $d U_{\text {err }}$ is the amount by which the elastic energy decays due to erratic motions of prevailing nonequilibrium associated polymer structures, living polymers in the case of the EHUT system. This is the process that gives rise to the first term in the right-hand side of Eq. (2). The released energy due to bond formation, $d U_{S}=-d U_{B}=\Phi d f$, has been split into a part $(1-\alpha) \Phi d f$ delivered as heat and a part $\alpha \Phi d f$ delivered as work done on the elastic component of the energy. Note that when bonds are formed, $d f$ is negative and $\Phi d f$ is positive. Clearly, we do not know how much of the released energy $\Phi d f$ is delivered as work and how much as heat. The second law of thermodynamics requires $(1-\alpha) \Phi d f$ to be large enough so that the corresponding increase of entropy outweighs the decrease of entropy resulting from the change of elastic energy (through a change of local strains). Within these limits, the elastic energy will increase by an amount $\alpha \Phi d f$. Near equilibrium, the second law of thermodynamics states that stress increases monotonically with increasing elastic 
energy. Since torque measurements with a rheometer are instantaneous, we conclude that the stress increases proportionally to $-d f$. In order to comply with the tensor character of the stress, the constant of proportionality has to transform as a tensor. The only tensor we can use is stress itself, leading to the contribution $-\sigma_{x y} B d f$ in Eq. (2).

Note that $B$ accounts for the combined effect of two different aspects of the relaxation process. First, we have assumed that bond formation can lead to work being done on the surroundings of two associating structural elements. Second, we have attributed a measurable shear stress increase to the locally increased elastic energy. In many situations this is actually not the case. Suppose that the bonding process leads to a system containing many randomly oriented elastically deformed domains. All offdiagonal components of the stress tensor will then on average be zero, much like the overall magnetization of a collection of magnetized Weiss domains is zero when they are randomly oriented. So a basic assumption underlying our equations is that the induced elastic domains are not isotropically oriented, but rather have an average orientation that gives rise to a nonzero contribution to the $x y$ component of the stress tensor.

In order to complete the model, we need constitutive equations for $r, \tau$, and $B$ in terms of $f$. For simplicity, and in order to limit the number of unknown parameters, we make the following choice:

$$
\begin{aligned}
r(f) & =r \\
\tau(f) & =\tau_{l}-\left(\tau_{l}-\tau_{s}\right) \frac{f}{f_{0}} . \\
B(f) & =b(1-f)^{-\kappa} .
\end{aligned}
$$

The first assumes that the relative rate with which new bonds are created is constant. Depending on the type of reaction, one might consider other forms here. The second is a simple linear interpolation in $f$ of the instantaneous relaxation time $\tau$ between a short time relaxation time $\tau_{s}$ and a long time relaxation time $\tau_{l}$. The reasoning behind the third expression is that what really matters for stress increase is the relative increase of the fraction of bonds materialized; in order to be more flexible we have changed the dependence on $(1-f)^{-1}$ into $(1-f)^{-\kappa}$, with $\kappa$ being a constant.

With this model we have fitted the experimental findings and plotted the results as drawn lines in Fig. 2. First of all, it is clear that the model, given its simplicity, captures the experimental data very well, with the only noticeable disagreement being near the stress minima. It is reassuring to notice that in all cases the positions of the maxima are well described.

The main reason why the model is able to describe the data is the fact that the stress evolving with time is the sum of two competing terms, where the first leads to a stress decrease while the second may lead to a stress increase depending on the sign of $B$. We have argued above that, depending on the interplay between shear induced order and bond formation, stress increase may be expected. The absolute values of both terms are decreasing with time, which does not imply, however, that they may never become equal. In order to clarify this point we combine Eqs. (1) and (2) to find $d \sigma_{x y}=-\sigma_{x y} d t / \tau_{\text {eff }}$, where $\tau_{\text {eff }}$ is an effective decay time given by

$$
\frac{1}{\tau_{\mathrm{eff}}}=\frac{1}{\tau}(1-B r f \tau)
$$

In the inset of Fig. 3 we have plotted $1 / \tau_{\text {eff }}$ as a function of time for systems A and C. Initially and at late times $\tau_{\text {eff }}$ is positive, giving rise to decaying stresses. At intermediate times $\tau_{\text {eff }}$ is negative, causing the stress to increase. At these times $B r f \tau$ is larger than one, implying that the natural stress decay cannot compete with the stress increase due to the formation of new bonds. Thus, stresses are increasing. When $t$ goes to infinity, $f$ goes to zero and therefore both $\tau$ and $\tau_{\text {eff }}$ go to $\tau_{1}$. The values of $1 / \tau_{1}$, obtained from the fits, are indicated as short drawn lines to the right of the inset in Fig. 3. It is interesting that the stress of the first system (A) decays exponentially in time soon after having reached its maximum, reflecting the Maxwellian relaxation of living polymers. The same does not apply in the other two cases, where the increase of the fraction of bonds is much slower. The stress decay after the maximum is described by a stretched exponential with exponents 0.54 for system B and

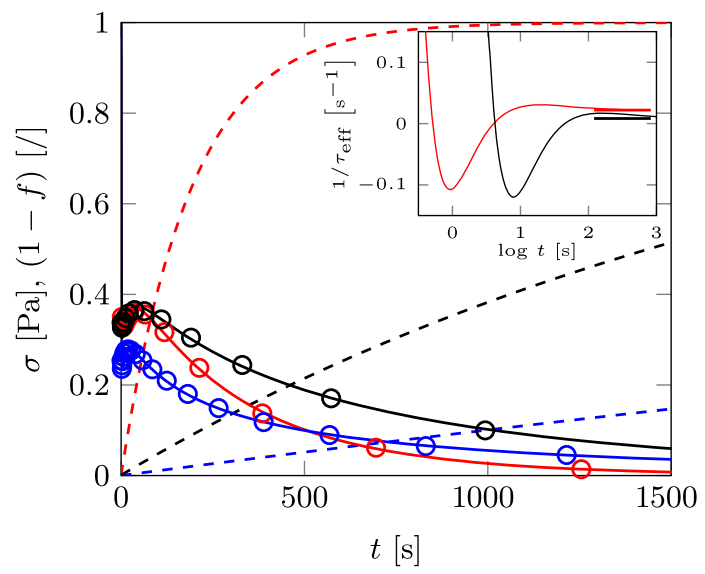

FIG. 3. Increase of fraction of formed bonds following flow cessation, $1-f$, with time along with the stress evolution for systems A, B, and C, respectively, in red, blue, and black at initial shear rates of 7,10 , and $4 \mathrm{~s}^{-1}$. The circles are experimental stress data, lines are the model fits, and dashed lines are the associated $1-f$ values. For system $\mathrm{A}$ in red, the time axis has been multiplied by 10 so that all data fit into the same plot. Inset: Variation of the inverse effective stress decay time $\left(\tau_{\text {eff }}\right)^{-1}$ with time (see text). Horizontal lines indicate the inverse decay times $1 / \tau_{1}$ to be reached at very long times. 
0.85 for system C. In Fig. 3 we plot the time dependence of both the stress and the fraction of bonds materialized in the system, $1-f(t)$. Note that the horizontal axis indicates seconds for systems $\mathrm{B}$ and $\mathrm{C}$, and tens of seconds for system A. As an aside, let us mention that the EHUT system stops showing a stress increase when the steady shear flow is prolonged to very high strain values. In this case the initially broken polymers start to reform during the shearing stage in a shear aligned state. After cessation of flow no new bonds are being created, and the system simply decays exponentially as a living polymer.

In all cases $\kappa$ was only slightly above one, while the initial value $f_{0}$ was only slightly below one. For the EHUT system this is not surprising. For the polymer systems this implies that the model in its present form is slightly oversimplified. In these cases, steady shearing has two effects. First, it will disrupt bonds leading to a decay of the bond density, as included in our model. $f=1$ does not refer to all bonds being broken, but to the maximum of bonds that can be broken by shearing the system. Second, depending on the total strain, it shifts the distribution of the remaining bonds over intramolecular and intermolecular bonds away from the corresponding equilibrium distribution. After all broken bonds have been reestablished, during the later part of the stress relaxation, the disturbed distribution of bonds must reequilibrate. This needs existing bonds to be broken and reestablish elsewhere. The timescale for this process must be set by an activation barrier of the order of the binding energy. This is only implicitly described in the present model.

The ingredients that a system must have in order to exhibit nonmonotonic stress relaxation after cessation of flow are twofold. First, the system should contain functional groups that are able to form bonds. The strength of the bonds must be such that the system accumulates elastic energy. Second, bond formation should be able to locally deform the material and induce stress. For the associative polymers this sets an upper limit to the bond densities. On the other hand, possible binding partners must meet before the order imposed by the shear has relaxed completely. In order to generate macroscopically measurable stresses, the stresses induced by the formation of bonds at different positions in the system must at least partly be aligned, as confirmed by the increase of birefringence (Fig. S3 of SM [19]).

In summary, we have provided experimental evidence for the unexpected nonmonotonic stress relaxation upon cessation of shear flow in different associating systems. Our novel findings are rationalized by a simple phenomenological model, which ensures thermodynamic consistency. The findings bear strong similarities to the polymerization of microtubules [24,25] (in particular with system A), which possess force generating mechanisms by performing work with energy provided by adenosine triphosphate (ATP) or guanosine triphosphate (GTP) [26], e.g., power strokes and ratchets [27], can heal defects [28], and are flow sensitive [29]. This sets the framework for further exploration and understanding of the physics of relaxation phenomena in synthetic and biological systems and paves the way for detailed structural experiments or simulations to exploit the possible anisotropic orientation of induced elastic domains.

We acknowledge support by the EU (FP7, Marie-Curie ITN, Grant No. 607937-SUPOLEN).

*These authors contributed equally to this work.
†dvlasso@iesl.forth.gr
${ }^{*}$ W.J.Briels@ utwente.nl

[1] Y. Serero, V. Jacobsen, J. F. Berret, and R. May, Macromolecules 33, 1841 (2000).

[2] L. Pellens, R. Gamez Corrales, and J. Mewis, J. Rheol. 48, 379 (2004).

[3] S. Suzuki, T. Uneyama, T. Inoue, and H. Watanabe, Macromolecules 45, 888 (2012).

[4] F. Meng, R. H. Pritchard, and E. M. Terentjev, Macromolecules 49, 2843 (2016).

[5] M. K. Sing, J. Ramirez, and B. D. Olsen, J. Chem. Phys. 147, 194902 (2017).

[6] M. Ballauff, J. M. Brader, S. U. Egelhaaf, M. Fuchs, J. Horbach, N. Koumakis, M. Krüger, M. Laurati, K. J. Mutch, G. Petekidis, M. Siebenbürger, T. Voigtmann, and J. Zausch, Phys. Rev. Lett. 110, 215701 (2013).

[7] E. Moghimi, A. R. Jacob, and G. Petekidis, Soft Matter 13, 7824 (2017).

[8] L. Mohan, R. T. Bonnecaze, and M. Cloitre, Phys. Rev. Lett. 111, 268301 (2013).

[9] A. Louhichi, Ph.D. thesis, University of Crete, 2017.

[10] J. Hendricks, Ph.D. thesis, KU Leuven, 2019.

[11] R. Fournier, Ph.D. thesis, Université Pierre et Marie Curie, 2019.

[12] J. K. G. Dhont, Phys. Rev. E 60, 4534 (1999).

[13] C. Pujolle-Robic, P. D. Olmsted, and L. Noirez, Europhys. Lett. 59, 364 (2002).

[14] J. Mewis and P. Moldenaers, Mol. Cryst. Liq. Cryst. 153, 291 (1987).

[15] G. Ducouret, C. Chassenieux, S. Martins, F. Lequeux, and L. Bouteiller, J. Colloid Interface Sci. 310, 624 (2007).

[16] T. Shikata, T. Nishida, B. Isare, M. Linares, R. Lazzaroni, and L. Bouteiller, J. Phys. Chem. B 112, 8459 (2008).

[17] L. Bouteiller, O. Colombani, F. Lortie, and P. Terech, J. Am. Chem. Soc. 127, 8893 (2005).

[18] J. Brassinne, A. Cadix, J. Wilson, and E. van Ruymbeke, J. Rheol. 61, 1123 (2017).

[19] See Supplemental Material at http://link.aps.org/ supplemental/10.1103/PhysRevLett.123.218003 for Fig. S2 and relevant discussion, which includes Refs. [20-23].

[20] A. Louhichi, A. R. Jacob, L. Bouteiller, and D. Vlassopoulos, J. Rheol. 61, 1173 (2017).

[21] M. Akbulut, N. K. Reddy, B. Bechtloff, S. Koltzenburg, J. Vermant, and R. K. Prud'homme, Langmuir 24, 9636 (2008). 
[22] G. G. Fuller, Optical Rheometry of Complex Fluids (Oxford University Press on Demand, New York, 1995).

[23] L. Pellens, J. Vermant, and J. Mewis, Macromolecules 38, 1911 (2005).

[24] A. Desai and T. J. Mitchison, Annu. Rev. Cell Dev. Biol. 13, 83 (1997).

[25] C. P. Brangwynne, F. C. MacKintosh, and D. A. Weitz, Proc. Natl. Acad. Sci. U.S.A. 104, 16128 (2007).
[26] S. Inoue and E. D. Salmon, Mol. Biol. Cell 6, 1619 (1995).

[27] A. Mogilner and G. Oster, Curr. Biol. 13, R721 (2003).

[28] L. Schaedel, K. John, J. Gaillard, M. V. Nachury, L. Blanchoin, and M. Thery, Nat. Mater. 14, 1156 (2015).

[29] K. Okeyoshi, R. Kawamura, R. Yoshida, and Y. Osada, Sci. Rep. 5, 9581 (2015). 